injection for SLNM of newly diagnosed EC undergoing surgical staging. The prima-ry endpoint of the study was to compare these two techniques in term of para-aortic detection rate.

Methodology This RCT included women with apparent stage I or II histologically confirmed endometrial cancer undergoing surgery were included in the study. Two groups were distinguished according to two different techniques of indocyanine green (ICG) sentinel lymph node mapping (SLNM): cervical versus hysteroscopic injection. This randomized trial was not blinded for both the patients and the surgeons.

Result(s)* Since March 2017 until April 2019, 165 patients were randomized: $85(51.5 \%)$ in the cervical group and 80 $(48.5 \%)$ in the hysteroscopic group. After randomization, 14 $(8.5 \%)$ patients were excluded from the study. Finally, 151 patients were included in the analysis: 82 (54.3\%) in the cervical group and $69(45.7 \%)$ in the hysteroscopic group. Hysteroscopy injection demonstrated a 10\% higher accuracy to detect SNLs in the paraaortic area compared to cervical injection, although this difference did not reach statistical significance. The hysteroscopic technique was better in detecting isolated SLN para-aortic $(5.8 \%$ vs $0 \%)$. Cervical injection was correlated with higher SLN detection rates at pelvic level compared to hysteroscopic injection. Pelvic and overall detection was superior in the cervical group.

Conclusion* The current study suggests the use of cervical injection rather than hysteroscopic injection due to its better identification of sentinel nodes (particularly in the pelvic area). Although, detection of SLN in the para-aortic area was slightly superior in patients undergoing a hysteroscopic injection, no significant difference with cervical injection was detected.

\section{IMPROVED PREOPERATIVE RISK STRATIFICATION IN ENDOMETRIAL CANCER: EXTERNAL VALIDATION OF THE ENDORISK NETWORK MODEL IN A POPULATION- BASED CASE SERIES}

${ }^{1} \mathrm{M}$ Grube*, ${ }^{2} \mathrm{C}$ Reijnen, ${ }^{3} \mathrm{PJ}$ Lucas, ${ }^{4} \mathrm{~F}$ Kommoss, ${ }^{5} \mathrm{FK}$ Kommoss, ${ }^{1} \mathrm{SY}$ Brucker, ${ }^{1} \mathrm{E}$ Oberlechner, ${ }^{1} \mathrm{~B}$ Krämer, ${ }^{1} \mathrm{~J}$ Andress, ${ }^{1} \mathrm{~F}$ Neis, ${ }^{6} \mathrm{~A}$ Staebler, ${ }^{7} \mathrm{JM}$ Pijnenborg, ${ }^{1} \mathrm{~S}$ Kommoss. ${ }^{1}$ Tuebingen University Hospital, Department of Women's Health, Tuebingen, Germany; ${ }^{2}$ Radboud university medical center, Department of Radiation Oncology, Nijmegen, Netherlands; ${ }^{3}$ University of Twente, Department of Data Science, Enschede, Netherlands; ${ }^{4}$ Institue of Pathology im Medizin Campus Bodensee, Friedrichshafen, Germany; ${ }^{5}$ Heidelberg University Hospital, Institute of Pathology, Heidelberg, Germany; ${ }^{6}$ Tuebingen University Hospital, Department of Pathology and Neuropathology, Tuebingen, Germany; ${ }^{7}$ Radboud university medical center, Department of Obstetrics and Gynaecology, Nijmegen, Netherlands

\subsection{6/ijgc-2021-ESG0.118}

Introduction/Background* Preoperative risk stratification of newly diagnosed endometrial carcinoma (EC) patients has been hindered by only moderate prediction performance for many years. Recently ENDORISK, a Bayesian network (BN) model using easily accessible biomarkers, showed increased predictive performance when compared to current guidelines. It was the aim of this study to validate ENDORISK by applying a locked-down model to a population-based case series of endometrial carcinoma patients.

Methodology We assessed a retrospective cohort of women from the Tuebingen University Women's Hospital surgically treated for EC from 2003-2013. Minimal requirements for using ENDORISK were: availability of preoperative tumour grade, at least 3 of ER, PR, p53 or L1CAM immunohistochemical biomarkers, at least 1 preoperative marker (PAP, CTscan, CA125 or thrombocyte count), pathologic examination of lymph nodes, and 5-year disease specific survival data (DSS). ENDORISK was applied and prediction accuracy of lymph node metastasis (LNM) as well as 5-year DSS was investigated. The model's overall performance was quantified by the Brier score, discriminative performance was measured based on the area under the curve.

Result(s)* A complete data set was evaluable from 247 patients. Median patient age was 64yrs (33-90), 78.1\% cases were endometrioid histotype. Grade distribution included 87 (35.2\%) G1, 106 (42.9\%) G2, and 54 (21.9\%) G3 tumours. $156(63.2 \%)$ patients had stage IA disease, with the remaining stage IB $(\mathrm{n}=52 ; 21.1 \%)$, stage II $(\mathrm{n}=12 ; 4.9 \%)$, and stage III/IV $(n=27 ; 10.9 \%)$. AUC for LNM prediction was 0.851 (95\% confidence interval [CI] 0.761-0.941) and 0.698 (95\% CI $0.595-0.800$ ) for 5-year DSS. The Brier scores were 0.06 for LNM and 0.09 for 5-year DSS, respectively. In 156 patients (63.2\%) LNM prediction was $\leq 5 \%$ (false-negative rate $0.6 \%$ ).

Conclusion* We have successfully demonstrated ENDORISK prediction of LNM and 5-year DSS in a large single-centre population-based cohort using preoperative clinical and biomarker data. Next steps will now have to focus on ENDORISK performance in clinical practice environments, e.g. dealing with missing data. Incorporating molecular profiling will be of key importance for future extended use. This external validation study reinforces previous findings and may support further promoting of data-based decision-making tools in EC research and patient care.

\section{CHARACTERISTICS AND PATTERNS OF CARE OF ENDOMETRIAL CANCER BEFORE AND DURING COVID- 19 PANDEMIC}

${ }^{1} \mathrm{G}$ Bogani ${ }^{*},{ }^{2} \mathrm{G}$ Scambia, ${ }^{3} \mathrm{~F}$ Landoni, ${ }^{4} \mathrm{~V}$ Zanagnolo, ${ }^{5} \mathrm{E}$ Sartori, ${ }^{6} \mathrm{~S}$ Cianci, ${ }^{6} \mathrm{~A}$ Ercoli, ${ }^{2} \mathrm{~F}$ Fanfani, ${ }^{7} \mathrm{R}$ Angioli, ${ }^{8} \mathrm{P}$ De laco, ${ }^{9} \mathrm{~A}$ Ciavattini, ${ }^{10} \mathrm{~L}$ Aguzzoli, ${ }^{11} \mathrm{~V}$ Remorgida, ${ }^{12} \mathrm{P}$ Benedetti Panici, ${ }^{13} \mathrm{G}$ Siesto, ${ }^{14} \mathrm{~V}$ Chiantera, ${ }^{15} \mathrm{E}$ Zupi, ${ }^{16} \mathrm{~A}$ Cianci, ${ }^{17} \mathrm{~T}$ Simoncini, ${ }^{18} \mathrm{~F}$ Raspagliesi* ${ }^{1}$ Fondazione IRCCS Istituto Nazionale dei Tumori; ${ }^{2}$ Policlinico Gemelli, IRCCS; ${ }^{3}$ University of Milan Bicocca; ${ }^{4}$ IEO; ${ }^{5}$ University of Brescia; ${ }^{6}$ University of Messina; ${ }^{7}$ Campus Biomedico Roma; ${ }^{8}$ University of Bologna; ${ }^{9}$ Università Politecnica delle Marche, Ancona; ${ }^{10}$ Azienda Unità Sanitaria Locale -IRCCS; ${ }^{11}$ University of Eastern Piedmont, Novara, Italy; ${ }^{12}$ La Sapienza di Roma; ${ }^{13}$ Humanitas University; ${ }^{14}$ University of Palermo; ${ }^{15}$ University of Siena; ${ }^{16}$ University of Catania; ${ }^{17}$ University of Pisa; ${ }^{18}$ Istituto Nazionale dei Tumori | Fondazione IRCCS

\subsection{6/ijgc-2021-ESGO.119}

Introduction/Background* COVID-19 outbreak has correlated with the disruption of screening activities and diagnostic assessments. The risk of delayed diagnosis has consequently increased during the pandemic. Endometrial cancer (EC) is one of the most common gynecological malignancies and it is often detected at an early stage, because it frequently produces symptoms. Here, we aim to investigate the impact of COVID19 outbreak on patterns of presentation and treatment of EC patients.

Methodology This is a retrospective study involving 54 centers in Italy. We evaluated patterns of presentation and treatment of EC patients before (period 1: 03/01/2019 - 02/29/2020) and during (period 2: 01/04/2020 - 3/31/2021) the COVID19 outbreak.

Result(s)* Medical records of 5,164 EC patients have been retrieved: 2,718 and 2,446 women treated in period 1 and 